\title{
Breaching the kinetic barrier to in vitro somatic stem cell propagation
}

\author{
Joshua R. Merok and James L. Sherley ${ }^{\dagger}$ \\ Division of Bioengineering and Environmental Health and Center for Environmental Health Sciences, \\ Massachusetts Institute of Technology, Cambridge, Massachusetts 02139, USA
}

\begin{abstract}
Here we have reviewed the conventional definitions and fundamental characteristics of the two basic types of stem cells, embryonic stem cells (ESCs) and somatic stem cells (SSCs). By taking into account the often-overlooked asymmetric cell kinetics of SSCs, we consider the evidence that should SSCs retain these growth kinetics in vitro, a natural kinetic barrier to SSC propagation exists. Recent discoveries showing that the tumor suppressor gene $p 53$ can act as a regulator of asymmetric cell kinetics provide a target pathway for in vitro SSC propagation strategies.
\end{abstract}

Early experiments by cell histologists such as Leblond inferred the existence of pluripotent progenitor cells, or stem cells $[1,2]$. As stem cell research progressed, it became apparent that there existed two basic types of stem cells (Figure 1). The first, ESCs, are derived from blastocysts and are sufficiently undifferentiated to allow them to give rise to any adult tissue [3]. This pluripotency can be seen in the ability of ESCs to be incorporated into developing mouse embryos during transgenic mouse production [4]. The other major type, SSCs, are stem cell populations found in specific adult tissues that are thought to divide indefinitely with restricted in vivo differentiation capacity [5]. SSC properties have classically been demonstrated by reconstitution of the ablated hematopoietic system of irradiated mice with a subpopulation of bone marrow or fetal liver cells [6].

The value of isolating and characterizing pure SSC populations is enormous. Already, current stem cell technology has yielded important disease therapies and research tools (e.g., bone marrow transplants and transgenic mice). Aside from gene therapy applications, an understanding of basic stem cell biology could have major impact in the fields of aging, development, and cancer. In addition, stem cell breakthroughs may provide the ideal building blocks needed for successful tissue engineering. While both ESCs and SSCs may be suited for these roles, studies with SSCs lack the moral and ethical dilemmas encountered in research with human ESCs [7]. Furthermore, the ideal future stem cell gene therapy applications should make use of autologous cells in order to minimize the risks of immunological rejection and disease contraction. Thus, the need for isolation and propagation of pure populations of SSCs is essential.

Most of the current SSC isolation and propagation strategies are based on a functional definition of SSCs that has been developed mostly from hematopoietic studies. These now classic bone marrow experiments-turned-treatments have

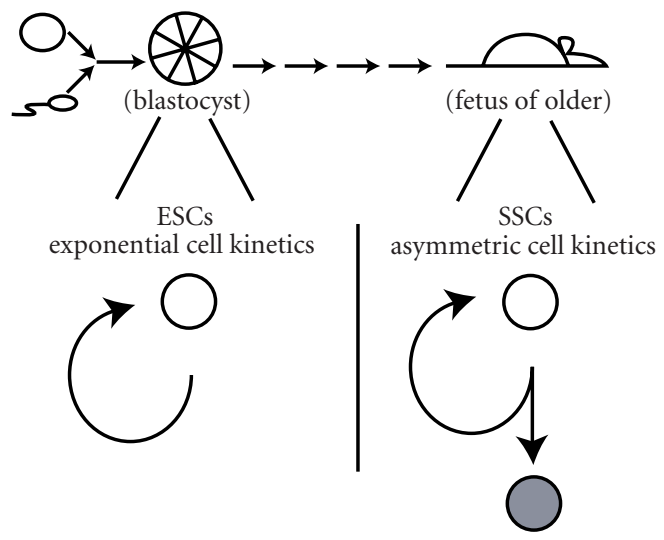

FIgure 1: ESCs versus SSCs. Two basic type of stem cells exist, the ESC and the SSC. ESCs are derived from blastocysts and divide with exponential cell kinetics. SSCs, however, are derived from somatic tissues and divide with asymmetric cell kinetics.

provided a concise functional definition of SSCs. Namely, convention now defines SSCs simply by scoring a cellpopulation's ability to replenish or regenerate the entire tissue of which it is a part. Despite caveats, such as performing experiments in lethally irradiated animals, this definition has thus far served researchers well. Using it, various fractionation and purification techniques (e.g., immunomagnetic separation and fluorescence-activated cell sorting) have been developed to greatly enrich for HSCs [8-10]. While the value of these particular cells for hematopoietic replacement therapy is unquestioned, the need for stem cells from other tissue systems is equally vital. Moreover, no known method currently used to enrich for HSCs has been able to purify them to homogeneity.

One major obstacle standing in the way of SSC isolations, such as HSCs, is the lack of a physical identifier for SSCs. Ide- 
ally, this physical identifier (e.g., a specific gene product or a set of gene products responsible for stem cell qualities) should be directly related to unique characteristics or qualities that are shared by all SSCs. Since no known cell-surface markers currently used to enrich for HSCs have been shown to provide a pure population of SSCs, [8-10] it seems worthwhile to consider other stem cell characteristics, in addition to tissue regeneration potential, to aid in the search for physical identifiers. One such characteristic is predicted to be asymmetric cell kinetics [11, 12].

In vitro cultures of immortal cells are commonly used in mammalian cell research. These cell lines, often transformed and typically harboring mutations in the $p 53$ tumor suppressor gene, grow with exponential kinetics [13]. This program, in which 1 cell begets 2,2 beget 4,4 beget 8 , and so on ad infinitum, is almost never found in the healthy somatic cells of adult mammals [14]. Instead, many adult somatic tissues undergo cell turnover in accordance with an asymmetric cell kinetics program, whereby stem cell division gives rise to (1) another stem cell and (2) a transit cell destined to produce a terminally differentiated lineage [14-16]. Once explanted, Rambhatla and others (this issue) postulate that tissue cells continue with a rudimentary form of this pattern in vitro. They propose that continued somatic stem cell kinetics in vitro results in the well-documented "senescence" behavior observed in many primary cultures $[17,18]$.

Now, with the suggestion that many primary cells divide with asymmetric kinetics, the past difficulties with SSC propagation in culture is not surprising. Even starting with a population of pure stem cells, transit cells are soon produced. Eventually, the culture flasks become replete and need to be passaged. By seeding a new flask with a portion of the old flask (standard cell culture technique), the total stem cell number per flask dwindles. This diluting-out of stem cells not only results in heterogeneous cultures, but it also sets up a selective pressure favoring cells with growth-activating mutations that disrupt the asymmetric cell kinetics program. This kinetic barrier to SSC propagation will have to be faced no matter what method of stem cell isolation is used, and it may ultimately be the major obstacle to maintaining wild-type stem cell strains in vitro.

In an attempt to understand the kinetic barrier to SSC propagation, Rambhatla and others have engineered cell lines that conditionally express a protein which is mutated in an astonishingly large number of immortalized cells-the tumor suppressor p53. The result has been cell lines that grow with inducible asymmetric cell kinetics. The implications, not only for the field of basic p53 research, but for actual SSC propagation in vitro are quite important. To be exact, $p 53$ expression has been shown to control asymmetric kinetics through control of inosine- $5^{\prime}$-monophosphate dehydrogenase (IMPD), the rate-limiting enzyme for guanine nucleotide synthesis $[19,20]$. As such, this regulatory pathway may be the key to controlling asymmetric kinetics in SSCs, thus ultimately allowing both the elucidation of physical identifiers of stem cells and the propagation of true wildtype SSCs in vitro.
In conclusion, although attempts to purify SSCs have been partially successful, no presently applied technology can isolate pure SSCs. With the present lack of a true physical identifier of SSCs, functional definitions, such as tissue reconstitution potential, must be used in purification strategies. One often-overlooked functional definition of SSCs is asymmetric cell kinetics. This functional definition leads to the important realization that even with a pure SSC population, in vitro propagation will be impossible without addressing the kinetic barrier posed by their unique cell kinetics program. Advances in understanding this kinetic barrier have been made by Rambhatla and others that point to the tumor suppressor $p 53$ (working through cellular guanine ribonucleotide pools) as an important regulator of asymmetric cell kinetics. Thus, engineered perturbations of this pathway may ultimately lead to successful SSC isolation and propagation in vitro.

\section{REFERENCES}

[1] Leblond CP, Walker BE. Renewal of cell populations. Physiol Rev. 1956;36:255-79.

[2] Leblond CP. Classical technics for the study of the kinetics of cellular proliferation. In: The Kinetics of Cellular Proliferation; 1959:31-47.

[3] Evans MJ, Kaufman MH. Establishment in culture of pluripotential cells from mouse embryos. Nature. 1981;292:154-156.

[4] Illmensee K, Mintz B. Totipotency and normal differentiation of single teratocarcinoma cells cloned by injection into blastocysts. PNAS. 1976;73(2):549-53.

[5] Loeffler M, Potten CS. Stem cells and cellular pedigrees-A conceptual introduction. In: C. S. Poetten, ed. Stem Cells. San Diego, CA;1997:1-28.

[6] Reisner Y, Itzicovitch L, Meshorere A, Sharon N. Hematopoietic stem cell transplantation using mouse bone marrow and spleen cells frationated by lectins. PNAS. 1978;75(6):2933-2936.

[7] Shapiro HT. Ethical dilemmas and stem cell research. Science. 1999;285:2065.

[8] Ziegler BL, Valtieri M, Porada GA, et al. KDR receptor: A key marker defining hematopoietic stem cells. Science. 1999;285:1553-1558.

[9] Gussoni E, Soneoka Y, Strickland CD, et al. Dystrophin expression in the $\mathrm{mdx}$ mouse restored by stem cell transplantation. Nature. 1999;401:390-394.

[10] Moore KA, Ema H, Lemischka IR. In vitro maintenance of highly purified, transplantable hematopoietic stem cells. Blood. 1997;89(12):4337-47.

[11] Cairns J. Mutation selection and the natural history of cancer. Nature. 1975;255:197-200.

[12] Thrasher JD. Analysis of Renwing epithelial cell populations. In: D. M. Prescott, ed. Methods in Cell Physilogy. Vol. 2. NY: Academic; 1966:323-357.

[13] Jia LQ, Osada M, Ishioka C, et al. Screening of p53 status of human cell lines using a yeast functional assay. Mol Carcinog. 1997;19(4):243-253. 
[14] Herrero-Jimenez P, Thilly G, Southam PJ, et al. Mutation, cell kinetics, and subpopulations at risk for colon cancer in the United States. Mutation Res. 1998;400:553578.

[15] Lajtha LG. Stem cell concepts. Differentiation. 1979;14:23-34.

[16] Potton CS. Cell cycles in cell hierarchies. Int J Radiat Biol. 1986;49:257-78.

[17] Hayflick L. The limited in vitro lifetime of human diploid cell strains. Exp Cell Res. 1965;37:614-36.

[18] Macierira-Coelho A, Azzarone B. Aging of human fibroblasts is asuccession of subtle changes in the cell cycle and has a final short stage with abrupt events.
Exp Cell Res. 1982;141:325-332.

[19] Sherley JL, Stadler PB, Johnson DR. Expression of the wild-type p53 antioncogene induces guanine nucleotide-dependent stem cell division kinetics. PNAS. 1995;92:136-140.

[20] Liu Y, Bohn SA, Sherley JL. Inosine-5' -monophosphate dehydrogenase is a rate-determining factor for p53dependent growth regulation. Mol Biol Cell. 1998;9:1528.

${ }^{\dagger}$ E-mail: jsherley@mit.edu 

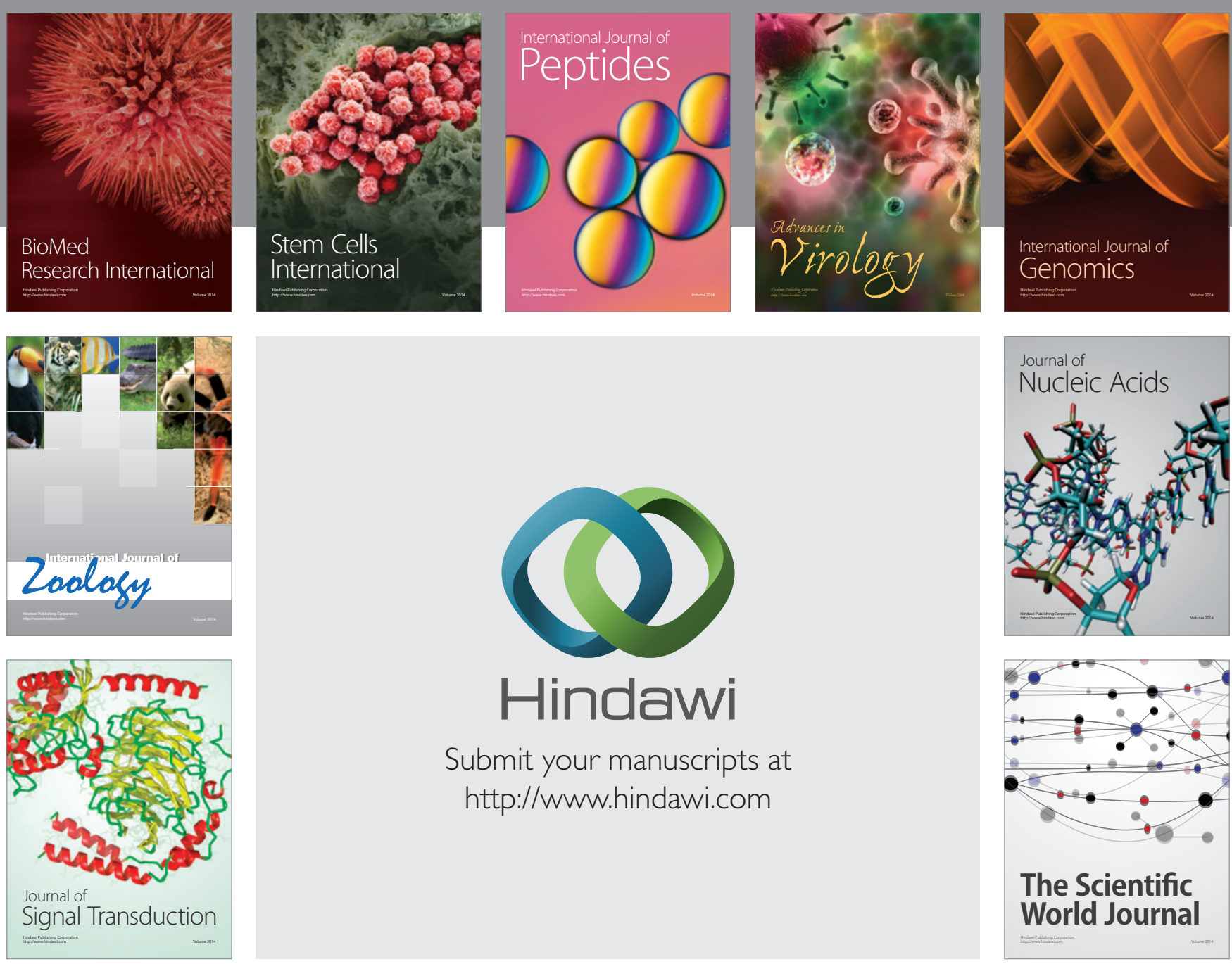

Submit your manuscripts at

http://www.hindawi.com
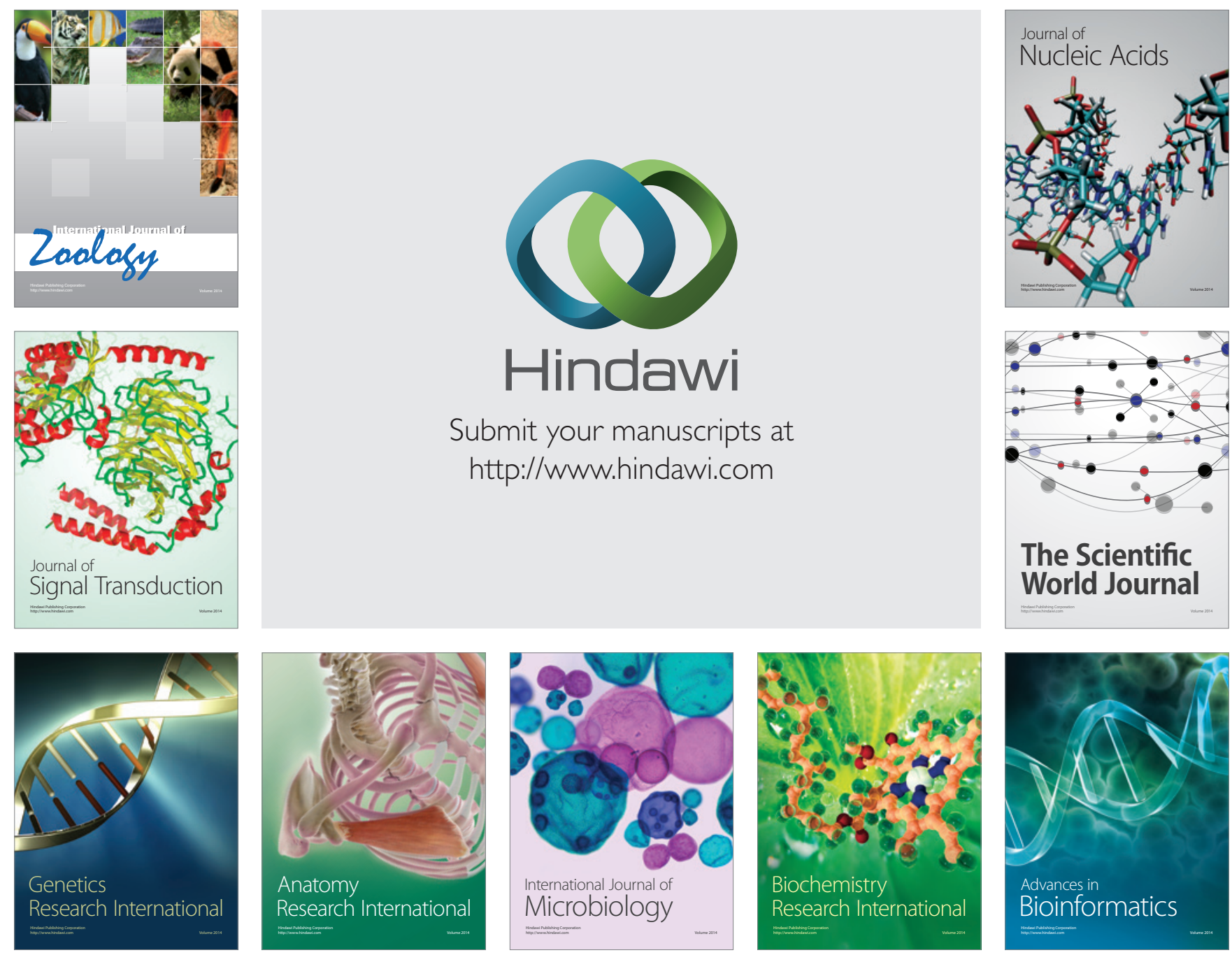

The Scientific World Journal
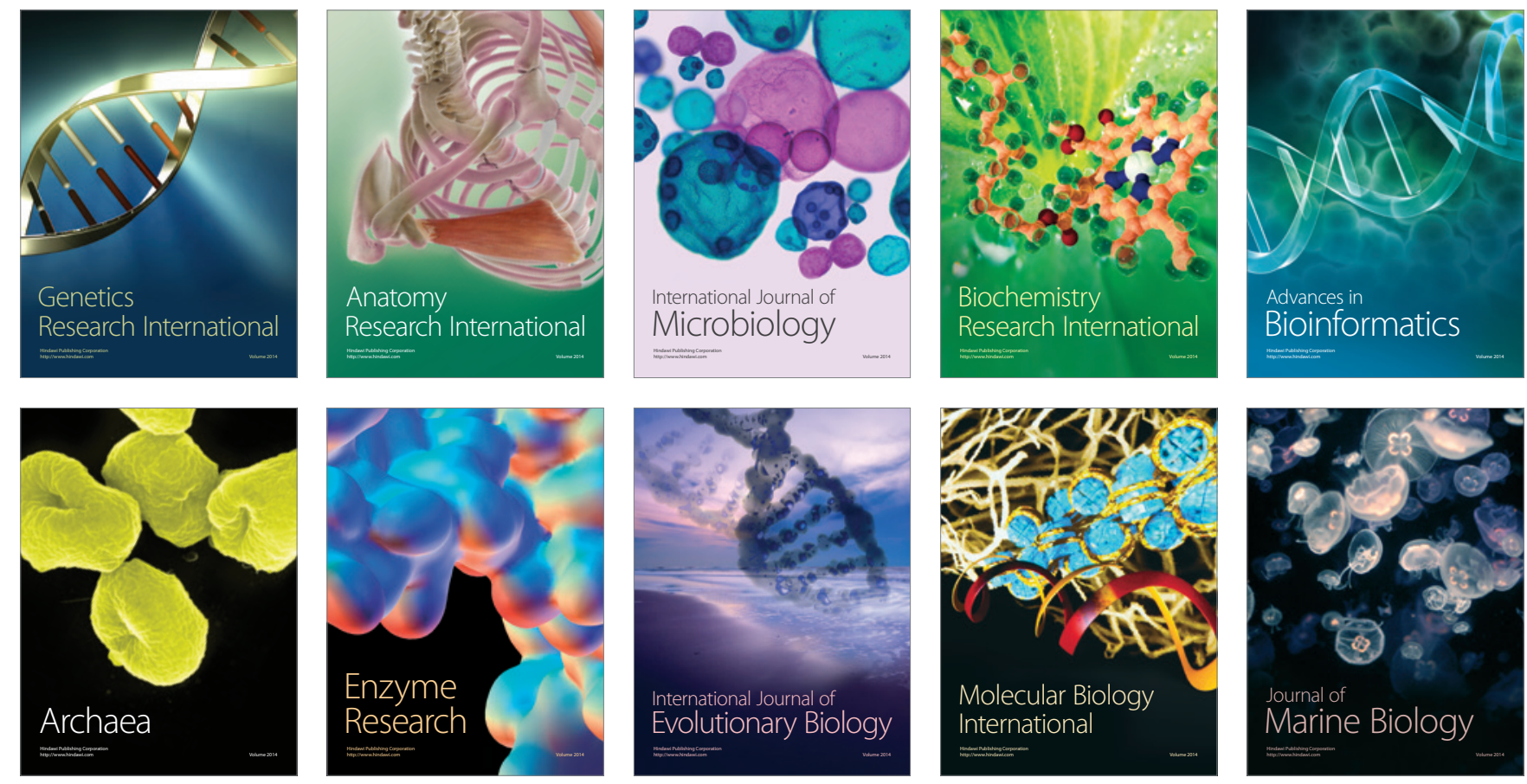